\title{
TUNABLE COLOR REFLECTOR WITH ZERO STATIC POWER
}

\author{
Mohsen Jafari ${ }^{*}$, L. Jay Guo ${ }^{l}$, and Mina Rais-Zadeh ${ }^{l, 2}$ \\ ${ }^{1}$ Department of Electrical Engineering, University of Michigan, Ann Arbor, Michigan, USA \\ ${ }^{2}$ NASA Jet Propulsion Laboratory (JPL), Pasadena, California, USA
}

\begin{abstract}
This paper introduces a tunable color reflector for use in low power display systems employing a phase change material (PCM) within a sub-wavelength layered structure. Optical index tunability of the PCM in the reflection spectrum permits vivid color tuning for low power applications. The phase change material used to achieve these results was Germanium Telluride (GeTe). Specifically, ultrathin film GeTe was sandwiched between a bottom metallic mirror and a top Indium Tin Oxide (ITO) layer. A thick phase-shift Silicon Dioxide $\left(\mathrm{SiO}_{2}\right)$ was placed under GeTe to enhance the color tunability in the setting of GeTe phase transition, thus improving color change. Low sensitivity to incident light angle and polarization provides the potential for this device to be very useful for portable device applications.
\end{abstract}

\section{INTRODUCTION}

Display systems and color filters are among the most commonly used optical components producing colors, where the core principle is based on emitting or filtering white light [1]. Active displays, which need constant power to produce a color, are well developed and utilized technology and have advanced considerably within the past decades [2]. Light emitting diodes (LED), which can produce an array of vivid colors, have both improved in quality and cost leading to their widespread use in commercial products, replacing the old liquid crystal display (LCD) technologies [3]. State of the art Organic LED (OLED) technology can address cost, power, refresh rate, and durability issues that were experienced with the use of traditional LCDs $[4,5]$. Despite such advancements in display technologies, active display systems remain to be the main source of power usage in electronic devices. Passive color displays that only consume power to switch color have almost zero static power and are efficient alternatives as ultra-low power displays, especially for low refresh rate applications [6].

Most of the modern display systems use three or more separate pixel components to produce a pixel of an image such as microelectromechanical (MEMS) based mirrors, color E-ink, OLED, etc. [7]. However, generating all three primary colors in a single (component) pixel has always been the best way to produce vivid images. An example of this is a single mirror interferometric device (SMI), where a moving MEMS mirror is used to tune the pixel color in the visible spectrum [8]. However, having movable objects has proven to be a major limitation of these devices in terms of durability, switching power consumption, and reliability in response to mechanical shocks in portable electronics where lower power is necessary. Structural-based color filters may be used but cannot provide the whole range of tunable colors. Chemical-based color pixels, where an electro-chromic, photo-chromic, or thermochromic material is used in a photonic cavity can provide a more versatile reflective display. Their tunability adds another dimension to the passive or active components at IR or microwave frequencies [9]. Furthermore, systems such as optical communication [10], memories [11], energy harvesters [12], as well as countless others, may benefit from tunable components to reduce process variation and improve stability when device parameters change in response to harsh environments or due to aging.

Liquid crystals [13], Germanium (Antimony) Telluride [14,
15], and Vanadium Dioxide $\left(\mathrm{VO}_{2}\right)$ [16] are among the most commonly used phase change materials as they exhibit significantly different refractive index values in their different phases. Using this mechanism, high amplitude modulation can be achieved by employing both real and imaginary parts of the refractive index [17]. Here, Germanium Telluride (GeTe) was used in a layered photonics structure to produce a color reflector as shown in Figure 1. High optical loss of GeTe in visible-near infrared (NIR) region must always be considered as a potential limitation for its use in transmission based devices [18]. This complication was avoided by making the GeTe very thin and utilizing a reflection-based device for ultra-low power displays [19]. As a result, two vivid colors (red and blue-purple) were achieved in two different crystallographic phases of GeTe. The devices have been switched more than 100 times with application of electrical pulses trains. Ultra-thin GeTe films used in these devices undergo a smoother, more complete, and more reliable crystallographic phase transition (Figure 2) with considerably decreased loss than what has been observed in devices with thicker films. An additional consideration is that unlike $\mathrm{VO}_{2}$ [16], both phases of GeTe are stable at room temperature, resulting in zero static power consumption. In other words, the devices consisting of GeTe are more stable during retention time without the need for constant heat pumping into the structure. Thus, if designed properly, GeTe-based devices would offer longer life-time with lower aging issues.

GeTe, even though a thermo-chromic PCM, could be transitioned electrically or optically, which may prove useful for a diverse field of applications with fully optical operation. Reliable, fast, and repeatable phase transitions are demonstrated for multiple devices showing minimum variation in their optical responses. Use of GeTe results in much higher stability due to its high phase changing temperature compared to $\mathrm{VO}_{2}$ and $\mathrm{GeSbTe}$. Compared to previous designs using a singfinale layer of PCM on a noble metal [19], use of optical cavity between PCM and the bottom reflector increases the modulation index per each nm of PCM. An antireflection thin ITO film on top is used to reduce the nonmodulated reflection and enhance the device dynamic color range.

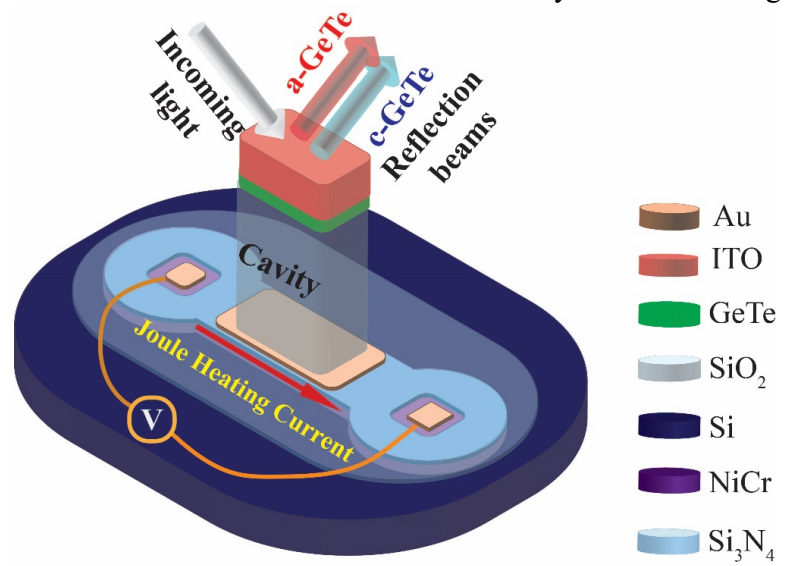

Figure 1. Schematic of the device consisting of a buried NiCr heater connected to gold electrodes to apply current. Stack of $\mathrm{TiO}_{2}$ (red) and $\mathrm{SiO}_{2}$ (blue) films is shown sitting on top of GeTe film in green. 


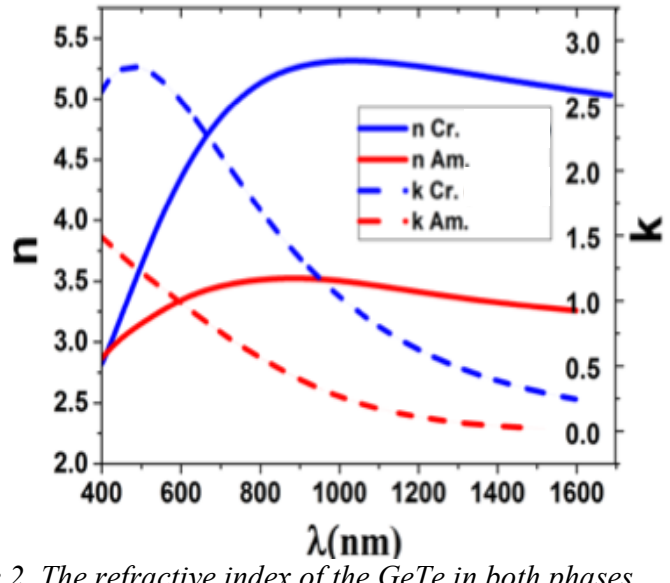

Figure 2. The refractive index of the GeTe in both phases.

The remaining sections of the paper follow this outline: design and characterization of the color filter; fabrication methods and phase transitioning; experimental results; discussion and conclusions.

\section{METHOD OF DESIGN AND FABRICATION \\ Theory and Design}

The device schematic shown in Figure 3(left) consists of a thin GeTe film $(20 \mathrm{~nm})$ sitting on top of $\mathrm{a} \mathrm{SiO}_{2}$ phase shift layer. This oxide layer serves as the body of the optical cavity to enhance the light reflection contrast. Under the oxide, a thick gold layer reflects all the light back to oxide. Compared to the thin film resonancebased shutter [18], where there is no optical cavity, addition of this phase shift improves the color contrast of the device in its two different states. This is crucial to achieve low loss high contrast performance with the thinnest possible PCM layer. The buried $\mathrm{NiCr}$ is located under the whole stack and used for phase transition through joule heating. To model the total light reflection of this device, one can use the transfer matrix method, where each layer's transfer matrix " $\mathrm{M}$ " is defined as:

$$
\mathrm{M}=\prod_{n}\left[\begin{array}{cc}
e^{\beta_{n}} & r_{n} e^{\beta_{n}} \\
r_{n} e^{-\beta_{n}} & e^{-\beta_{n}}
\end{array}\right] .
$$

Here, $r_{n}$ is the light reflection from $n^{\text {th }}$ interface and $\beta_{n}$ is the phase accumulation in the $\mathrm{n}^{\text {th }}$ layer, which are both defined as:

$$
\begin{aligned}
& \mathrm{r}_{\mathrm{n}}=\left(\frac{k_{n}-k_{n+1}}{k_{n}+k_{n+1}}\right) . \\
& \beta_{\mathrm{n}}=\mathrm{e}^{i k_{n} t_{n}} .
\end{aligned}
$$

where $\mathrm{k}_{\mathrm{n}}$ is wavevector in layer $\mathrm{n}$ and $\mathrm{t}_{\mathrm{n}}$ is $\mathrm{n}^{\text {th }}$ layer thickness. Wavevector $\left(k_{n}\right)$ is $n_{n} \times \cos (\theta)$ for s-polarization and $n_{n} / \cos (\theta)$ for $p$ polarization with $\theta$ being the light incidence angle. One can mathematically derive total light reflection from expanding eq (1) as:

$$
R=\left|\begin{array}{l}
M_{01} \\
M_{00}
\end{array}\right|^{2}
$$
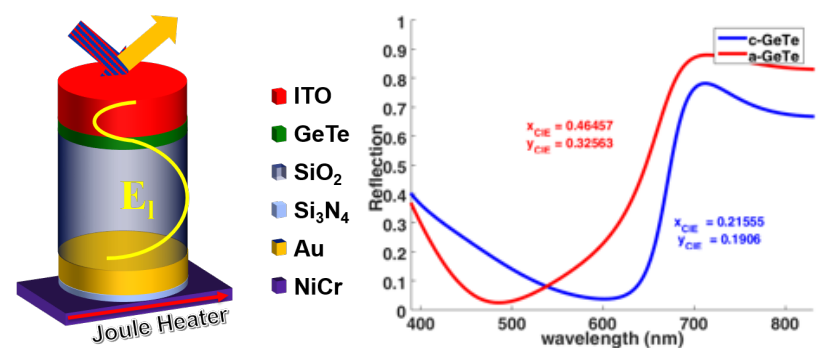

Figure 3. (left) Multi-layer DBR based shutter using ultra-thin PCM layer $(20 \mathrm{~nm})$. (right) Light reflection for both phases of the structure (inset: CIE standard coordinates).

Figure 3 demonstrates the device structure consisting of (from top): antireflective ITO coating; GeTe PCM layer; $\mathrm{SiO}_{2}$ phase shift layer; bottom gold reflector; $\mathrm{SiN}_{3}$ passivation layer; buried $\mathrm{NiCr}$ heater. Placing highly absorptive crystalline GeTe film with thickness $t_{G T}$ in between low loss $\mathrm{SiO}_{2}$ and ITO films generates a node in the structure for electromagnetic waves. Bottom gold acts as a node in the reflecting waves since the traveling waves have to have similar amplitude which can only satisfy the boundary condition when they are both zero. If the light wavelength is twice the distance between the gold mirror and the lossy crystalline GeTe film, it can reflect back almost perfectly from the stack as shown with reflection peaks at $700 \mathrm{~nm}$. The position of peak is readily derivable as:

$$
\frac{\lambda_{P}}{2}=2 \times k \times t_{S i O 2} \times n_{S i O 2} \sim 500 * 1.45=725 \mathrm{~nm}
$$

Tuning of the peak wavelength within the visible wavelength range follows Equation (5) for $\mathrm{SiO}_{2}$ changing from $150 \mathrm{~nm}$ to $300 \mathrm{~nm}$. However, light will acquire minimum loss and phase shift for a round trip between top ITO and bottom gold. Addition of propagation loss through ITO, $\mathrm{SiO}_{2}$, each of $\mathrm{t}_{\mathrm{GT}} / 2$ crystalline $\mathrm{GeTe}$ film, and reflection loss in gold surface results in imperfect reflection at $700 \mathrm{~nm}$. This reflection peak does not move when GeTe transitions to the amorphous state, due to high loss of amorphous $\mathrm{GeTe}$ at $700 \mathrm{~nm}$. Moreover, the wide reflection minimum is due to dispersion of refractive indices for gold and GeTe in the visible region. As shown in Figure 2, GeTe refractive index changes when it goes through crystallographic phase transitions between its two stable phases. This phase transition tunes the dispersion of its refractive index, which causes different outcomes for the total light reflection. This is due to tuning in the reflection coefficient in ITO/GeTe and $\mathrm{GeTe} / \mathrm{SiO}_{2}$ interfaces. Materials with different refractive indices, if used instead of ITO and $\mathrm{SiO}_{2}$, will result in different reflection spectra and thus different colors. To quantitatively show the color change the International Commission on Illumination (CIE-1931) standard is being used. Using density functions defined in this standard, one can transfer the device reflection spectrum to a set of coordinates ( $\left.\mathrm{x}_{\mathrm{CIE}}, \mathrm{y}_{\mathrm{CIE}}\right)$. Locating such coordinates on a colored map, similar to Figure 4, shows the true reflected color of the device. This device even with an ultra-thin PCM film $(\sim 20 \mathrm{~nm})$ tunes the "xCIE" and "yCIE" coordinates by $\sim 53 \%$ and $42 \%$, respectively, when GeTe layer is transitioned from amorphous to crystalline state. This change is enough to change the purple reddish color of the device to dark blue (shown in Figure 4). 


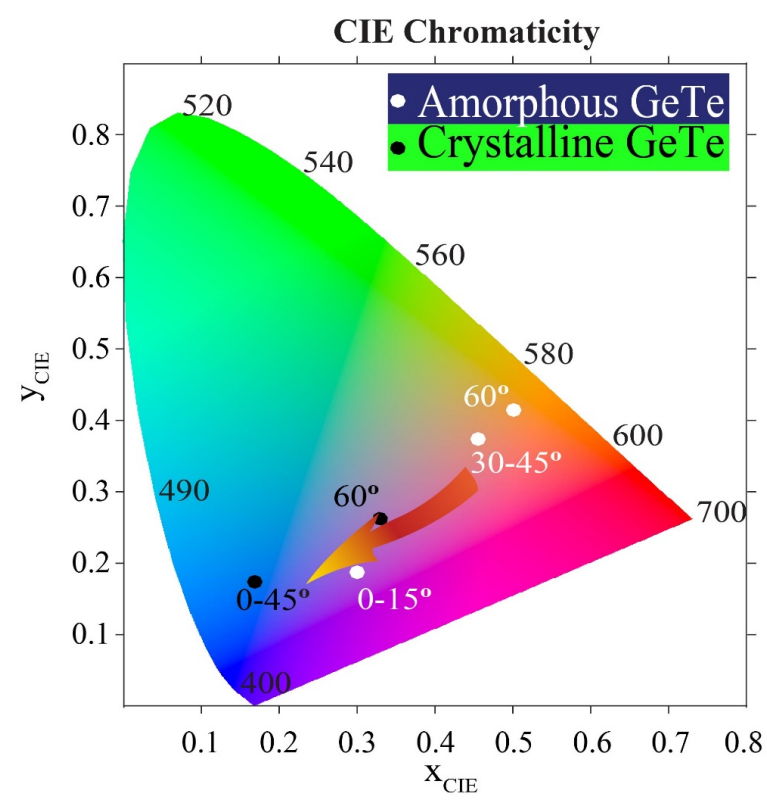

Figure 4. CIE chromaticity graph showing device color in different incidence angles and for crystalline (black dots) and amorphous (white dots) GeTe.

\section{Angle and polarization dependence}

Device colors do not change significantly and remain relatively similar at normal incidence even up to $45^{\circ}$ oblique incidence. This is demonstrated in Figure 4 for both phases with the inset showing the corresponding colors at each angle.

\section{FABRICATION AND PHASE TRANSITION}

\section{Fabrication:}

Fabrication starts with cleaned single side polished silicon wafers. The bottom joule-heater is then patterned using conventional optical lithography and the lift-off process. For this purpose, different refractory metals are tested to match input impedance to that of the DC probes used to apply heating pulses. $\mathrm{NiCr}$ showed the most reliability with most number of switching cycles for the device. A thin $10 \mathrm{~nm} \mathrm{Si}{ }_{3} \mathrm{~N}_{4}$ is deposited on the heater to isolate it electrically from the cavity's bottom reflector which is gold in this case. After passivation, gold mirror is deposited and patterned using the same process used for $\mathrm{NiCr}$ heater. The stack is then annealed at $200{ }^{\circ} \mathrm{C}$ to release any residual stress between interfaces. This enhances the yield and reduces the risk of film delamination during next the high temperature Plasma Enhanced Physical Vapor Deposition (PEVCD) step. $\mathrm{SiO}_{2}$ is then deposited using PECVD as the cavity body using Plasma-Therm 790 PECVD tool (Plasma-Therm Co.) at $200^{\circ} \mathrm{C}$ at a rate of $7.5 \mathrm{~A} / \mathrm{sec}$. GeTe films with different thicknesses are then deposited at room temperature in a magnetron deposition system from Kurt J. Lesker ${ }^{\circledR}$ company from a 3 inch $\mathrm{Ge}_{50} \mathrm{Te}_{50}$ target (Mitsubishi Material Co.) as the top cavity reflector. Disposition rate was maintained at a rate of $0.66 \mathrm{~A} / \mathrm{sec}$ keeping the rate of $\mathrm{Ar}^{+}$carrier bombardment with $60 \mathrm{~W} \mathrm{RF}$ power. This controlled ultra-low rate deposition results in a more uniform film with better adhesion to the bottom $\mathrm{SiO}_{2}$ layer. Moreover, assputtered amorphous GeTe has lower optical loss in visible and NIR wavelengths. $\mathrm{SiO}_{2}$ films were then deposited. A controlled etching process was developed to dry-etch GeTe film into smaller sizes. This step was followed by ITO sputtering at room temperature using DC reactive sputtering in the same sputtering chamber. A dry-etch was then used to etch through the whole stack and access the bottom heater to apply the joule-heating pulse for GeTe phase transitions.

\section{Phase Transition}

Two of the main challenges in the color reflector design process were the design of the heater and the selection of a material with proper resistivity and thermal stability. $\mathrm{NiCr}$, which is a thermally stable material with resistivity of $150 \times 10^{-8} \Omega \mathrm{m}$, was used as the heater adjacent to GeTe. Being isolated from the reflecting gold, the $\mathrm{NiCr}$ heater provides the heat required for phase transition of GeTe through joule heating pulses applied to the pads in each side of the device. Pulse energy for crystallization was measured to be $80 \mathrm{~nJ}$ with outstanding low current of $40 \mathrm{~mA}$.

\section{EXPERIMENTAL RESULTS}

Reflection measurements were performed using a Woollam M2000 spectroscopic ellipsometer (JA Woollam Co) at different angles ranging from $45^{\circ}$ to $75^{\circ}$ due to the limitation of the tool setup. Samples were annealed at different temperature ranging from 165 ${ }^{\circ} \mathrm{C}$ to $180{ }^{\circ} \mathrm{C}$. The temperature was controlled using an IRthermometer. The reflection of the device for both " $s$ " and " $p$ " polarizations are shown in Figure 6 at different temperatures at $55^{\circ}$ (left) and $65^{\circ}$ (right). The GeTe and $\mathrm{SiO}_{2}$ film thicknesses for these devices were $20 \mathrm{~nm}$ and $250 \mathrm{~nm}$ respectively. Both polarizations show a wide mode at $\sim 740 \mathrm{~nm}$ and a relatively narrower mode at $\sim 380 \mathrm{~nm}$ verifying the cavity optical response. The wavelength of these two modes is almost independent of the GeTe phase due to the high loss of both amorphous (a)-GeTe and crystalline (c)-GeTe in the visible wavelengths. The reflection dip for $p$ polarization, on the other hand is tunable depending on the GeTe phase. Shown in this figure, GeTe crystallization causes a blue shift in the p polarized light reflection dip. This blue shift results in better coupling to the mode at $380 \mathrm{~nm}$, i.e. higher light reflection at that wavelength. This along with slight blue shift of the mode at 740 $\mathrm{nm}$ due to higher effective refractive index of the cavity yield to a bluer color to reflect from the device. To easily compare the device color tuning during a crystallization event, light reflection at RGB wavelengths are summarized in Table 1 from amorphous phase to fully crystalline films.

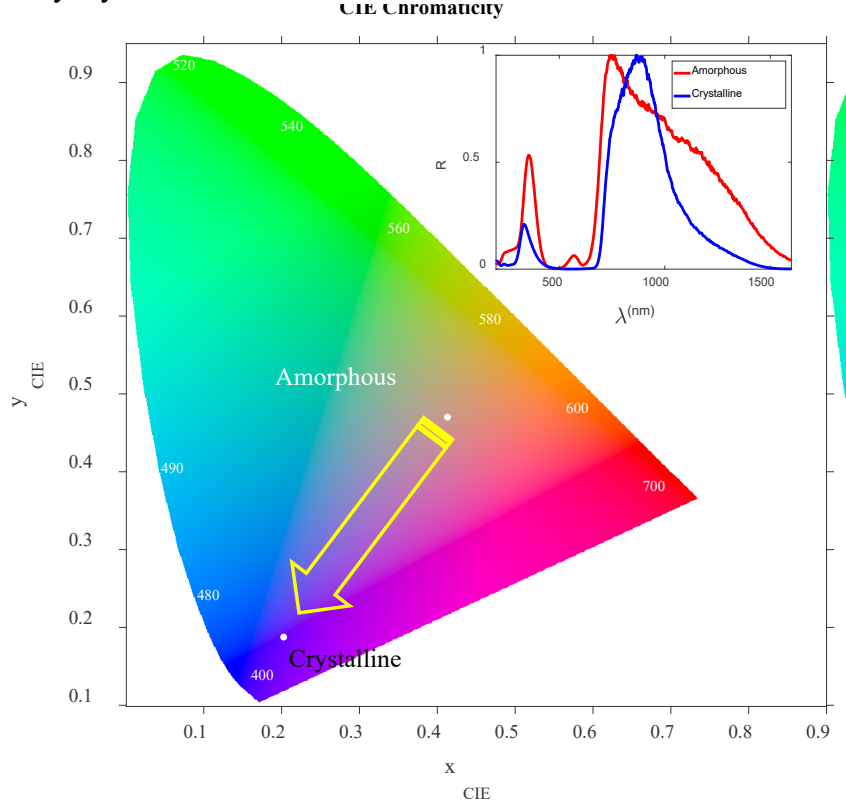

Figure 5. ON and OFF states reflection spectra for non-polarized light using spectroscopic ellipsometer at $45^{\circ}$ incident angle. Both states were measured at room temperature in ambient light. 
Device reflection is normalized using the sensitivity transfer function of human eyes' cone cells to replicate the true reflected color. The reflection spectrum after normalization is shown in (inset). Such a reflection is then normalized by the CIE standard to find the exact reflected color of the device (Figure 5). Furthermore, the actual color of the device within their pixel areas are shown Figure 7 under ambient white light illumination.

Table 1. Device average reflection change at visible wavelengths under white ambient light

\begin{tabular}{|c|c|c|c|}
\hline$\lambda_{\text {avg }}$ & Color & $\overline{\frac{\boldsymbol{R}_{\text {partial-cryst }}}{\overline{\boldsymbol{R}_{\text {amorphous }}}}}$ & $\overline{\overline{\boldsymbol{R}_{\text {full-cryst }}}}$ \\
\hline 470 & Blue & 0.9688 & 1.8801 \\
\hline 530 & Green & 1.016 & 0.405 \\
\hline 700 & Red & 1.02 & 1.08 \\
\hline
\end{tabular}
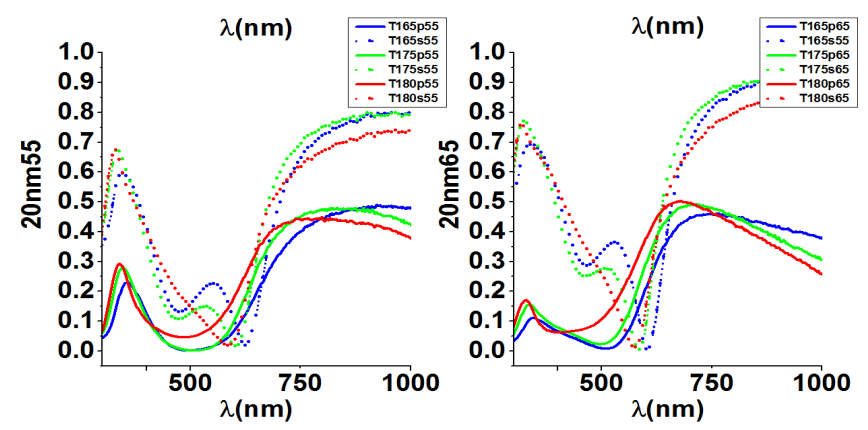

Figure 6. Device reflection at different temperature from $165{ }^{\circ} \mathrm{C}$ to $180^{\circ} \mathrm{C}$ for both $s$ and p polarization. The results shown for $55^{\circ}$ and $65^{\circ}$ incidence angles.
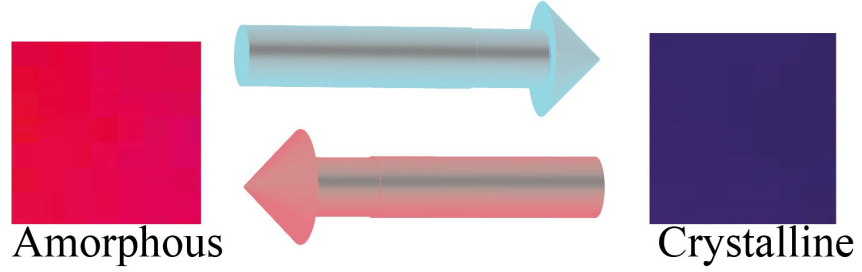

Figure 7. Devices actual colors as they are seen under white ambient light at $45^{\circ}$ degree for amorphous (left) and crystalline (right) phases of GeTe.

\section{CONCLUSION}

In this paper, a tunable color reflector was introduced using a phase change material (GeTe) sandwiched between a top ITO antireflective coating and a bottom gold mirror. Adding a $250 \mathrm{~nm}$ thick $\mathrm{SiO} 2$ phase shift layer between gold and thin PCM resulted in nodes in the structure and almost perfect reflection. This phase shift layer also helped to increase the color contrast created by GeTe phase transition. Design and modeling details were discussed along with the fabrication method. The device color was change from reddish purple to dark blue by employing a $20 \mathrm{~nm}$ film thickness of GeTe PCM. Without losing the generality, the same idea could be expanded to achieve multi-color performance with increasing the numbers of the PCM layers if an effective transition method is used to selectively switch each layer separately.

\section{ACKNOWLEDGEMENT}

The authors thank the staff at the Lurie Nanofabrication Facility, University of Michigan, Ann Arbor, MI, USA for providing fabrication facilities. This work is supported by University of Michigan

\section{REFERENCES}

[1] Yokogawa, Sozo, Stanley P. Burgos, and Harry A. Atwater. "Plasmonic color filters for CMOS image sensor applications." Nano Letters 12.8 (2012): 4349-4354.

[2] Zeng, Beibei, Yongkang Gao, and Filbert J. Bartoli. "Ultrathin nanostructured metals for highly transmissive plasmonic subtractive color filters." Scientific reports 3 (2013): 2840.

[3] Tan, Zhi-Kuang, et al. "Bright light-emitting diodes based on organometal halide perovskite." Nature nanotechnology 9.9 (2014): 687-692.

[4] Salehi, Amin, et al. "Highly Efficient Organic Light-Emitting Diode Using A Low Refractive Index Electron Transport Layer." Advanced Optical Materials 5.11 (2017).

[5] Page, Zachariah A., et al. "Novel Strategy for Photopatterning Emissive Polymer Brushes for Organic Light Emitting Diode Applications." ACS central science 3.6 (2017): 654-661.

[6] Yan, Jin, et al. "A full-color reflective display using polymerstabilized blue phase liquid crystal." Applied Physics Letters 102.8 (2013): 081102.

[7] Ma, Ji. "Advanced MEMS-based technologies and displays." Displays 37 (2015): 2-10.

[8] Hong, John, et al. "Continuous color reflective displays using interferometric absorption." Optica 2.7 (2015): 589-597.

[9] Vafapour, Zohreh, et al. "Graphene-based mid-infrared biosensor." JOSA B 34.12 (2017): 2586-2592.

[10] Nafari, Mona, and Josep Miquel Jornet. "Modeling and performance analysis of metallic plasmonic nano-antennas for wireless optical communication in nanonetworks." IEEE Access 5 (2017): 6389-6398.

[11] Ríos, C., Stegmaier, M., Hosseini, P., Wang, D., Scherer, T., Wright, CD., Bhaskaran, H. and Pernice, W.H., "Integrated allphotonic non-volatile multi-level memory." Nature Photonics, 9(11), pp.725-732. (2015)

[12] Nafari, A., and H. A. Sodano. "Surface morphology effects in a vibration based triboelectric energy harvester." Smart Materials and Structures 27.1 (2017): 015029.

[13] Humar, M., et al., "Electrically tunable liquid crystal optical microres- onators," Nature Photonics, 3(10), pp. 595-600. (2009).

[14] Hosseini, P., Wright, CD., and Bhaskaran, H., "An optoelectronic framework enabled by low-dimensional phasechange films". Nature, 511(7508), pp. 206-211. (2014)

[15] Jafari M. and Rais-Zadeh M., "Zero-static-power phase-change optical modulator," Optics letter, pp. 1177-1180. (2016).

[16] Wang H., et al. "Fabrication of vanadium oxide micro-optical switches," Sens. and Act. A, pp. 108-112. (2005).

[17] Jafari, Mohsen, and Mina Rais-Zadeh. "An ultra-high contrast optical modulator with $30 \mathrm{~dB}$ isolation at $1.55 \mu \mathrm{m}$ with $25 \mathrm{THz}$ bandwidth." In Photonic Fiber and Crystal Devices, vol. 10382, p. 1038211. SPIE, 2017.

[18] Jafari, M., and Rais-Zadeh, M. "A 1550 NM phase change electro-optical shutter." Micro Electro Mechanical Systems (MEMS), 2016 IEEE 29th International Conference on. IEEE, 2016.

[19] Jafari, M., Guo, LJ., and Rais-Zadeh, M., "An ultra-fast optical shutter exploiting total light absorption in a phase change material." In Proc. of SPIE Vol, vol. 10100, pp. 101000I-1. (2017).

[20] Jafari, M., Guo, LJ., and Rais-Zadeh, M., " Tunable color reflector with zero static power " In review. 